\title{
It's easy being green
}

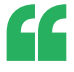

The challenge now involves finding a strategy to use these nanoparticles

in a way that will reveal useful diagnostic or functional information about the gut

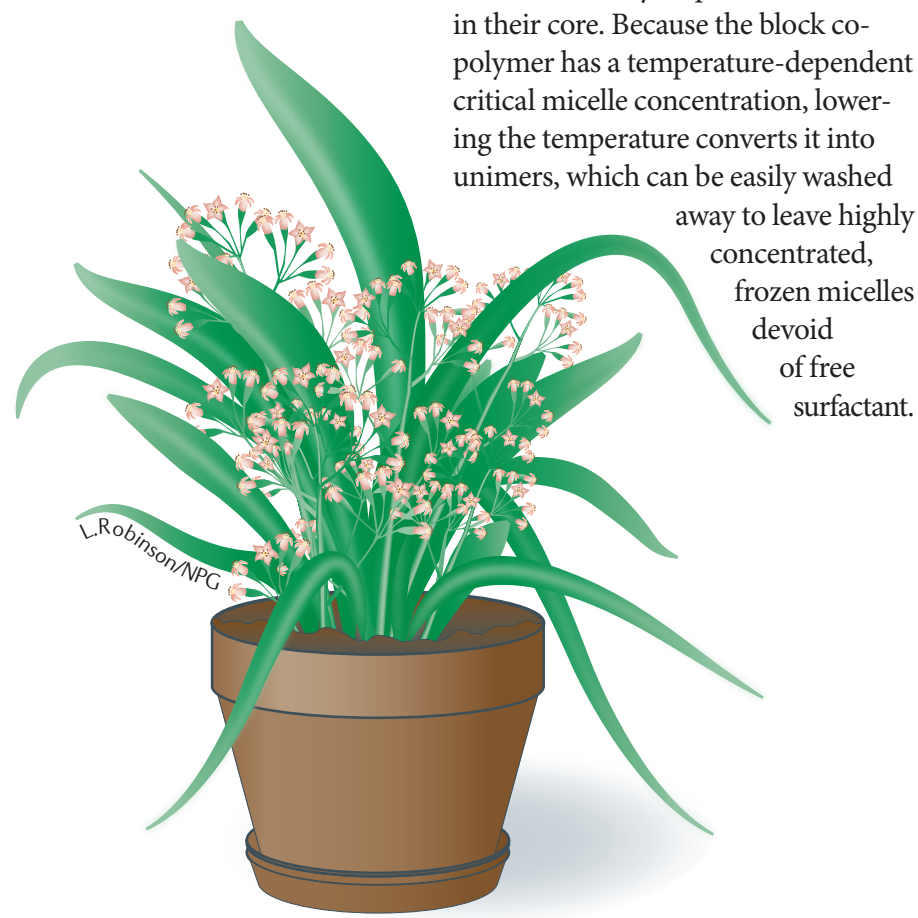

This approach can be used to To better diagnose and treat gastrointestinal disorders, non-invasive multimodal imaging techniques are needed. However, despite the successful application to other organs and regions of the body, multimodal imaging of the gut is limited by a lack of suitable contrast agents. Owing to a promising new class of materials surfactant-stripped frozen micelles - this may soon be set to change.

Reporting in Advanced Materials, Jonathan Lovell and co-workers describe the preparation of surfactant-stripped nanoscale frozen pheophytin (that is, demetallated chlorophyll) micelles that are active for at least three important imaging modalities. The strategy to prepare frozen micelles was reported by Lovell's team in 2014. Briefly, a hydrophobic molecule is dissolved in organic solvent then added to an aqueous surfactant (a block copolymer) solution in which micelles form with the hydrophobic molecule in their core. Because the block copolymer has a temperature-dependent critical micelle concentration, lowering the temperature converts it into away to leave highly micelles of free urfactant. prepare formulations of hydrophobic active pharmaceutical ingredients and contrast agents that are otherwise difficult to administer in sufficiently high doses. In addition to having activity for several imaging modalities, an ideal gut contrast agent must be safe and have high stability in the intestine and gut. Previously, the group demonstrated activity for two imaging modalities photoacoustic imaging and positron emission tomography - of the intestine using frozen micelles of naphthalocyanine dyes. However, owing to self-quenching, the hydrophobic dyes were unsuitable for fluorescence imaging, which is a cheap, fast, good-resolution and thus highly desirable modality. Moreover, naphthalocyanine is not currently approved for use in humans.

The main physical shortcoming of the hydrophobic dyes, namely, their unsuitability for fluorescence imaging, was overcome in this work. Chlorophyll is an ideal candidate fluorescent molecule as it forms a part of all human diets. Although the researchers observed that frozen chlorophyll micelles lost all fluorescence activity and were not highly stable, they found that the demetallated form, pheophytin, is more hydrophobic than chlorophyll, which renders it more suitable for the surfactant-stripping method. Surprisingly, the pheophytin micelles were shown in vitro to be highly stable under the harsh conditions of the gut, displaying no loss of optical activity. "The surfactant-stripped pheophytin micelles maintained a degree of near-infrared fluorescence so that they could be used for fluorescence imaging. We are not exactly sure of the reason for this, but suspect it might have to do with the pigment packing in the micelles," explains Lovell. In addition to recording the fluorescence distribution in the intestine, the pheophytin micelles were successfully used for functional photoacoustic imaging and positron emission tomography (after chelation with ${ }^{64} \mathrm{Cu}$ radioisotope).

In a mouse model, the nanoparticles were found to be completely excreted after 24 hours with biodistribution analysis showing no accumulation in the main organs. Moreover, the nanoparticles showed no toxicity either towards human epithelial colorectal adenocarcinoma cells or from histological analysis. "Pheophytin is consumed orally every day in human diets - any green food you eat contains chlorophyll. We believe that these nanoparticles should be safe to consume since there is minimal excipient in the nanoparticles; they are formed from an edible component; and they are not absorbed into the body at all but pass safely and completely through the gastrointenstinal tract," says Lovell.

From a materials perspective, the system is very simple to use and for manufacturing, scale up would likely be relatively straight-forward. "The challenge now," explains Lovell, "involves finding a strategy to use these nanoparticles in a way that will reveal useful diagnostic or functional information about the gut and developing a plan for clinical translation."

\section{Adam Brotchie}

ORIGINAL ARTICLE Zhang, Y.et al. Surfactantstripped frozen pheophytin micelles for multimodal gut imaging, Adv. Mater. http://dx.doi.org/10.1002/ adma.201602373 (2016)

FURTHER READING Zhang, Y. et al. Therapeutic surfactant-stripped frozen micelles, Nat. Commun. 7,11649 (2016) | Zhang, Y. et al. Non-invasive multimodal functional imaging of the intestine with frozen micellar naphthalocyanines, Nat. Nanotechnol. 9, 631-638 (2014) 